\title{
La structuration de la desserte militaire sur le réseau PLM (1862-1936)
}

Thomas Bourelly

\section{(2) OpenEdition \\ Journals}

Édition électronique

URL : https://journals.openedition.org/rhcf/515

DOI : 10.4000/rhcf.515

\section{Éditeur}

Rails \& histoire

\section{Édition imprimée}

Date de publication : 1 novembre 2006

ISSN : 0996-9403

Référence électronique

Thomas Bourelly, « La structuration de la desserte militaire sur le réseau PLM (1862-1936) », Revue d'histoire des chemins de fer [En ligne], 35 | 2006, mis en ligne le 01 juin 2011, consulté le 22 avril 2022 URL : http://journals.openedition.org/rhcf/515; DOI : https://doi.org/10.4000/rhcf.515

Ce document a été généré automatiquement le 22 avril 2022

Tous droits réservés 


\title{
La structuration de la desserte militaire sur le réseau PLM (1862-1936)
}

\author{
Thomas Bourelly
}

1 L'utilisation des chemins de fer par les autorités militaires est connue en ce qui concerne les périodes de conflits et les transports de troupes ${ }^{1}$. Mais la dimension « industrielle » des rapports entre le chemin de fer et l'administration de la Guerre aux $\mathrm{xix}^{\mathrm{e}}$ et $\mathrm{xx}^{\mathrm{e}}$ siècles a été peu étudiée ${ }^{2}$. Le cas des embranchements particuliers militaires sur le réseau PLM permet de considérer cette dimension sous un angle différent. Destinés à mettre les établissements commerciaux et industriels en relation avec les voies de chemin de fer, les embranchements représentent un domaine particulier au sein du monde ferroviaire. Leur usage s'est répandu durant la seconde moitié du xix ${ }^{\mathrm{e}}$ siècle. Ces infrastructures ont d'abord attiré l'attention des ingénieurs et des juristes ${ }^{3}$ et, si l'historiographie militaire fait beaucoup référence au chemin de fer, notamment la Revue du génie militaire, elle s'intéresse peu au rôle des embranchements particuliers. Pourtant, une des rares études sur ces voies ferrées publiées dans la Revue générale des chemins de fer est l'œuvre d'un militaire ${ }^{4}$. L'intérêt de ces dispositifs en matière d'histoire locale, technique et économique est néanmoins reconnu. Analysé dans sa thèse par François Caron ${ }^{5}$, ce mode d'approvisionnement n'a, depuis lors, jamais fait l'objet d'une attention particulière, mis à part quelques travaux récents ${ }^{6}$. À l'instar de l'étude menée par Jean-Pierre Williot, qui souligne l'importance des embranchements ferroviaires dans l'industrie gazière parisienne ${ }^{7}$, l'analyse des relations entre la Compagnie PLM et les autorités militaires permet de retracer la mise en place d'un dispositif de desserte stratégique autour des lignes reliant Paris à la Méditerranée, entre 1862 et 1936.

2 Constitué en 1857, le réseau PLM offre un exemple intéressant du fait de son étendue et de sa situation, en retrait par rapport aux frontières du Nord et de l'Est. De grands centres militaires sont situés le long de ses lignes et notamment dans la ville de Lyon, cœur du réseau et principale place de l'arrière durant la Première Guerre mondiale. La 
structuration d'un réseau d'embranchements militaires entre 1862 et 1936 peut être analysée par le biais des projets de traités approuvés par le conseil d'administration de la Compagnie $\mathrm{PLM}^{8}$. Cette dernière conclut avec les autorités de l'armée différents accords au sujet de raccordements particuliers, en vue du transport de marchandises en provenance ou à destination d'établissements militaires. Traditionnellement, les principaux bénéficiaires de la desserte ferroviaire sont les secteurs métallurgique, chimique et charbonnier, grands pourvoyeurs de trafic, mais sur le réseau PLM le client principal demeure l'État. Près de $10 \%$ des traités d'embranchement approuvés par le conseil d'administration concernent la desserte de diverses administrations publiques : Postes et Télégraphes, Commerce, Finances, Manufactures, Ponts et Chaussées et surtout les ministères de l'Air, de la Marine et de la Guerre. Ces trois dernières administrations représentent $71 \%$ des conventions passées avec l'État. Près de 7,5\% des traités conclus par la Compagnie PLM entre 1862 et 1936 concernent la desserte d'établissements militaires: arsenaux, poudrières, forges de la marine, magasins, hangars, dépôts ou centres d'aviation'.

Contrairement aux secteurs miniers et métallurgiques, qui bénéficient de raccordements dès la création du chemin de fer dans les années 1830, l'autorité militaire tarde à reconnaître l'importance d'une desserte efficace. Pourtant l'armée conçoit assez tôt l'intérêt du chemin de fer, comme en témoignent dès 1847 les essais pour le transport de troupes de cavalerie sur le chemin de fer entre Paris et Corbeil. Il faut attendre la guerre de 1870 pour que soit mise en évidence cette nécessité de raccordement, afin d'assurer un approvisionnement rapide et efficace. Un effort est alors engagé dans ce but : un tissu ferroviaire au service de l'administration militaire se structure progressivement.

Du conflit de 1870-1871 à celui de 1939, il convient donc d'analyser comment les ministères de la Guerre, de la Marine et de l'Air prennent conscience de l'importance capitale de la desserte de leurs établissements et comment ils envisagent le rôle du chemin de fer d'une manière nouvelle. Il faut tout d'abord comprendre comment s'associent les éléments industriels et militaires après la crise de 1870-1871. Par la suite, l'étude de l'évolution vers un dispositif de desserte entièrement au service de la Guerre, entre 1914 et 1919, permet de déterminer comment les embranchements militaires hérités du premier conflit mondial confèrent un dynamisme nouveau au réseau PLM durant la période de l'entre-deux-guerres.

\section{L'association des éléments industriels et militaires}

\section{La prise de conscience des lacunes}

5 Les autorités militaires conçoivent rapidement l'importance des chemins de fer et perçoivent ce nouveau de mode de transport comme un outil révolutionnaire ${ }^{10}$. Néanmoins la précocité de cette prise de conscience est sans comparaison avec l'exemple britannique ${ }^{11}$. En France, la première utilisation militaire des chemins de fer date de la guerre de Crimée (1854-1855). Les différentes compagnies assurent alors le transport des troupes vers les ports d'embarquement de Marseille et Toulon. Quelques années plus tard, en 1859, la Compagnie PLM est chargée d'assurer l'acheminement des soldats vers la frontière lors de la campagne d'Italie. Or ce type de transport concerne principalement les troupes et présente des caractéristiques différentes de celles du 
trafic commercial, nécessitant une organisation minutieuse. À cet égard, une réflexion autour des transports militaires s'engage le 15 mars 1869, suite à la création par le maréchal Niel d'une commission centrale des chemins de fer. Chargée d'étudier diverses questions, cette commission composée de civils et de militaires insiste notamment sur «la nécessité de raccorder aux gares les principaux établissements militaires, arsenaux, magasins et établissements de manutention $»^{12}$. À la mort du maréchal Niel, la même année, le maréchal Lebœuf, nouveau ministre de la Guerre, abandonne le programme de réformes et les mesures préconisées ne sont pas appliquées.

6 Le conflit de 1870-1871 est alors marqué par de graves difficultés d'approvisionnement sur les réseaux ferrés. L'encombrement des voies et des gares rend le service difficile, voire impossible. Pour la période qui suit le conflit, 7500 wagons chargés d'approvisionnements sont recensés sur l'unique réseau de $\operatorname{Lyon}^{13}$. Le seul rôle reconnu aux raccordements ferroviaires sur le réseau PLM durant ce conflit est d'avoir permis la fuite de Garibaldi et de son état-major au moyen de wagonnets abandonnés sur l'embranchement des mines d'Épinac, en Bourgogne ${ }^{14}$.

Dénonçant la situation générale et s'appuyant sur le gain de temps et d'argent offert par la voie ferrée, Prosper Jacqmin, ingénieur des Ponts et Chaussées et directeur de l'exploitation des Chemins de fer de l'Est, demande dès 1872 la reprise des projets de la commission Niel. Désirant associer l'élément militaire à l'élément technique, il souhaite « relier les arsenaux et les établissements militaires au réseau général des chemins de fer, partout où cette jonction n'existe pas encore $»^{15}$.

Dans le cas de la Compagnie PLM, malgré l'existence d'établissements militaires d'envergure le long du réseau, tels que les arsenaux de Lyon et Toulon, il faut attendre ces années 1870 pour que soient autorisés les premiers embranchements. Pourtant, dès 1859, des habitants du quartier Perrache, à Lyon, font remarquer que « dans l'intérêt de l'État, la Compagnie PLM eut dû établir un embranchement à destination de l'important arsenal depuis longtemps $»^{16}$.

9 On peut s'interroger sur le désintérêt du ministère de la Guerre envers ce moyen d'approvisionnement. L'absence de traités entre les compagnies de chemin de fer et les autorités militaires paraît surprenante dans la mesure où l'armée semble capable de leur assurer un trafic important. Cette lacune en matière de desserte est d'autant plus criante que les principaux fournisseurs de l'armée ont intégré depuis plusieurs années le chemin de fer comme outil d'approvisionnement. À l'instar du secteur métallurgique, principal client de la Compagnie PLM, les industries d'armement font rapidement preuve de modernité. Les principaux partenaires de l'armée se situent à proximité des bassins houillers, notamment le long de la ligne de Saint-Étienne à Lyon, sur laquelle l'usage des embranchements particuliers s'est développé depuis les années 1830. Mentionnées par François Crouzet, il s'agit des sociétés Jacob Holtzer à Firminy, des aciéries de Saint-Étienne ou de Schneider et Cie, raccordées durant les années 1860 et pour certaines héritières de voies établies antérieurement ${ }^{17}$.

\section{Établir des liens étroits}

L'année 1872 voit le premier raccordement du ministère de la Guerre sur le réseau PLM. La manufacture d'armes de Saint-Étienne, créée en 1764, est le premier établissement à faire l'objet d'un traité d'embranchement ${ }^{18}$. La compagnie conclut par la suite de 
nombreux actes similaires avec les administrations de la Guerre et de la Marine, 29 entre 1872 et 1890 (fig. 1). Tous les établissements militaires sont intéressés par la desserte ferroviaire: hangars et dépôts de matériel, magasins, parcs à fourrages, poudrières, forges et arsenaux. Des centres importants se structurent autour du rail, comme à Clermont-Ferrand, avec l'embranchement de l'artillerie dit des "Gravanches ", à Dijon avec les magasins des subsistances militaires et un arsenal, à Besançon, Moulins et Nevers, où sont desservis divers magasins d'approvisionnement, ainsi qu'à Lyon, qui accueille un parc d'artillerie, des magasins militaires, un parc à fourrages et un arsenal. Ce dernier établissement, plus tard dénommé atelier de construction de Lyon, symbolise à lui seul le paradoxe de la desserte militaire. Installé dès 1848 à proximité du point d'arrivée de la ligne de Saint-Étienne à Lyon, il n'est raccordé au chemin de fer que 28 ans plus tard.

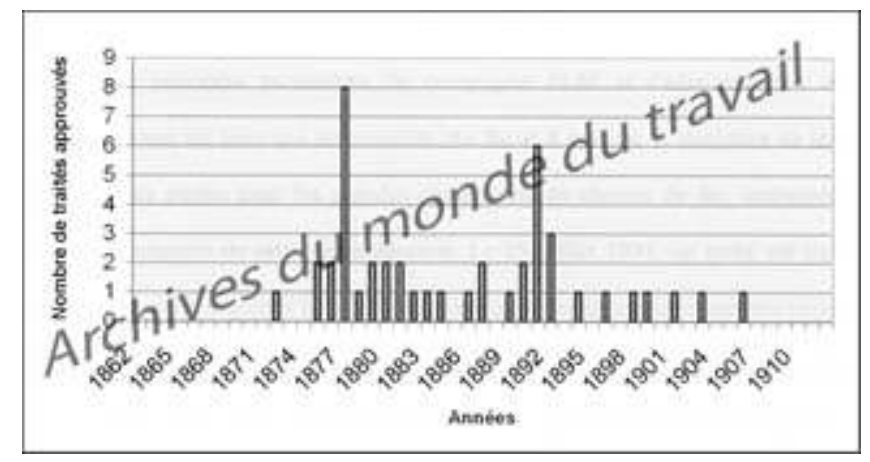

Figure 1. Traités d'embranchement conclus entre la Compagnie PLM et le ministère de la Guerre (1862-1912). Source : procès-verbaux du conseil d'administration de la Compagnie PLM, avril 1862 avril 1913, AN (CAMT), 77 AQ 180 à 203.

11 Devenus de véritables partenaires, la Compagnie PLM et l'administration militaire cherchent à affermir les liens qui les unissent. Progressivement, le ministère de la Guerre noue des rapports étroits avec les grandes compagnies de chemin de fer, désireuses de conserver les transports de cette administration. Le 15 juillet 1891, un traité est signé : les réseaux s'engagent à transporter la totalité du matériel et des approvisionnements appartenant au département de la Guerre. De son côté, l'armée s'engage à donner aux compagnies la totalité de ses transports, s'interdisant notamment le trafic par voie navigable ${ }^{19}$. La Compagnie PLM approuve la même année six traités d'embranchements particuliers, et trois l'année suivante. Les principaux centres militaires s'assurent de nouvelles conditions de desserte, comme à Nevers ou Dijon, tandis que de nouveaux établissements sont desservis avec les stations-magasins d'Auxerre, Montereau et Nuits-sous-Ravière. Les séquelles de la défaite de 1871 sont encore vives et l'autorité militaire, consciente du rôle du chemin de fer, évite d'établir des raccordements dans les régions frontalières ${ }^{20}$. Le ministère de la Guerre privilégie des zones plus en retrait, desservies par le réseau PLM. L'armée obéit alors à une conception centralisatrice de l'espace ferroviaire, autour de points stratégiques.

12 Un partenariat privilégié est institué entre les réseaux de chemins de fer et l'administration militaire. Néanmoins, avant la convention de 1891, la primauté du transport par rail est généralement assurée par la simple construction d'un embranchement. C'est le cas de l'arsenal de Lyon qui, dès la création de son raccordement en 1876, voit ses approvisionnements assurés par le chemin de fer et non par la voie navigable ${ }^{21}$. Établi le long de la Saône sur un terrain de $16000 \mathrm{~m}^{2}$, cet établissement est plusieurs fois étendu et les ateliers de construction rassemblent en 
18991245 personnes $^{22}$. Si l'on peut difficilement quantifier l'apport des établissements militaires trafic, l'envergure de certains d'entre eux laisse penser que le tonnage transporté devait être important.

13 Suite à la convention de 1891, le ministère de la Guerre est donc devenu un client majeur. Le conseil d'administration de la Compagnie PLM approuve 16 traités entre 1891 et 1906, preuve des relations étroites nouées entre les deux parties. Conscient de la nécessité d'une desserte efficace, le ministère de la Guerre est soucieux de l'entretien et de l'amélioration de ses embranchements. Le quart des traités approuvés durant cette période concerne la réfection et la réparation de voies, leur allongement ou la pose d'installations nouvelles, telles que des plaques tournantes, au sein des établissements desservis. L'administration militaire assume entièrement tous les frais de réparations et ne bénéficie d'ailleurs d'aucun avantage sur les prix de construction et d'entretien de ses embranchements. Ainsi, malgré les engagements de 1891, le ministère de la Guerre est considéré comme un client à part entière, au même titre que les représentants du commerce et de l'industrie.

\section{La ruée vers le rail}

Hormis les contrats passés directement avec la Compagnie PLM pour la desserte de ses établissements, l'autorité militaire use avantageusement de son droit de regard sur les constructions nouvelles. Des commissions mixtes de travaux publics, composées de membres du génie et des Ponts et Chaussées, sont chargées d'examiner tous les projets d'établissement de lignes et d'infrastructures nouvelles. Dès qu'une voie ferrée emprunte ou longe un terrain militaire, une conférence est ouverte en présence d'officiers du génie. Parfois ces derniers saisissent cette opportunité pour imposer certaines conditions et s'assurer d'une desserte gratuite en cas de nécessité. En 1891, statuant sur la demande d'un industriel de Vénissieux, dans le Rhône, pour traverser une enceinte fortifiée, le colonel Rousset, directeur du génie à Lyon, obtient le droit d'utiliser en cas de mobilisation la voie et le matériel de transport du demandeur, ainsi que les bâtiments situés le long de la voie ferrée et pouvant servir de magasins ${ }^{23}$.

Si l'administration de la Guerre recherche les avantages offerts par les chemins de fer, elle n'envisage pas uniquement le raccordement de ses établissements aux grands réseaux. Dans le département du Rhône, les voies ferrées secondaires (autres compagnies d'intérêt général, chemins de fer d'intérêt local et tramways) sont sollicitées. Le fort de Vancia, à proximité de Lyon, est raccordé dès 1875 aux voies de la Compagnie du chemin de fer des Dombes, reliant Sathonay à Bourg ${ }^{24}$. Par ailleurs, par convention du 15 décembre 1891, les véhicules de l'arsenal de Lyon sont autorisés à utiliser les voies de la Compagnie des omnibus et tramways de Lyon, afin de relier une annexe de cet établissement ${ }^{25}$. Enfin, en 1896, les docks du magasin militaire de Villeurbanne sont reliés à la gare de la Compagnie de l'est de Lyon, elle-même reliée par embranchement au réseau PLM ${ }^{26}$. L'administration de la Guerre cherche donc à exploiter au maximum les possibilités offertes par la proximité des chemins de fer ou des voies de tramways, tout en mettant à profit son droit de servitude, en vue de diversifier ses moyens de desserte et d'assurer pour ses établissements un meilleur fonctionnement, en prévision des conflits à venir.

Si le tissu d'embranchements particuliers à destination des établissements de la Guerre n'est pas totalement structuré au début du $\mathrm{XX}^{\mathrm{e}}$ siècle et si les raccordements militaires 
ne remplissent aucune fonction en matière de défense stratégique et de transport de troupes, leur rôle dans la guerre totale est pourtant prépondérant.

\section{Le chemin de fer au service de la guerre totale}

\section{La préparation au conflit}

17 À la veille du premier conflit mondial, le tissu d'embranchements militaires autour des lignes PLM n'est pas complètement achevé. On ne recense aucun traité entre 1906 et 1913. Face à la montée des tensions politiques, les autorités militaires cherchent à optimiser la trame ferroviaire existante et demandent dès 1912 à l'administration des chemins de fer d'effectuer des travaux d'intérêt stratégique. Outre les infrastructures nécessaires à l'embarquement de troupes, la mise en place des courants de circulation et l'amélioration des installations de traction, la Compagnie PLM porte ses efforts sur les embranchements desservant des établissements de la Guerre : stations-magasins de Besançon, Dijon, Châlon, Nevers, arsenaux de Lyon et Clermont-Ferrand, poudrerie du Bouchet $^{27}$. La compagnie étudie ou réalise l'extension de nombreuses voies particulières. Le montant des travaux militaires exécutés sur le réseau durant les exercices 1912, 1913 et le premier semestre de 1914 s'élève à plus de quatre millions de francs. Par ailleurs, les services du génie, de l'artillerie et de l'intendance adressent diverses demandes de matériel en vue de la construction de voies desservant leurs installations. La valeur du matériel livré par la Compagnie PLM, principalement des rails et des traverses, est estimée à 110000 francs $^{28}$. À la veille du conflit, le dispositif de desserte particulière a pris forme.

Si les exigences techniques sont respectées, les conditions d'utilisation des embranchements exploités ou réquisitionnés par les services militaires restent à définir. La convention entre le ministère de la Guerre et les réseaux de chemins de fer en date du 20 octobre 1915 permet la mise en vigueur de conditions d'exploitation particulières, de manière à résoudre les difficultés survenues suite à la réquisition des réseaux le 31 juillet $1914^{29}$. Il est établi que l'usage des embranchements par le département de la Guerre ne doit connaître aucune restriction au niveau de la fréquence des manœuvres :

19 "Le service sera assuré sans autres limites que celles qui résultent des possibilités techniques et de la nécessité de sauvegarder la sécuritée ${ }^{30}$. »

20 En échange de ces facilités d'utilisation, l'administration de la Guerre renonce à contester l'application des tarifs résultant du cahier des charges des compagnies. Cet accord prévoit en outre les difficultés à venir dans l'exploitation des embranchements et instaure un tarif moindre en cas de séjour prolongé du matériel sur les voies. Par ailleurs, les réseaux appréhendent les dépenses de manœuvres supplémentaires pouvant être générées par le conflit et fixent une taxe spécifique. Les principes exposés dans cet accord vont régir les conventions passées entre l'administration militaire et la Compagnie PLM durant le conflit.

21 Le cadre réglementaire d'exploitation mis en place, l'ouverture d'embranchements nécessaires à l'effort de guerre peut être envisagée. Or, depuis le 31 juillet 1914, les réseaux sont placés sous autorité militaire et l'application des accords du 15 juillet 1891 est suspendue. La préparation des traités nouveaux est laborieuse et ce n'est que fin décembre 1916 que la compagnie commence à recevoir des approbations de l'état- 
major. Les autorisations d'embranchements particuliers relèvent du $4^{\mathrm{e}}$ bureau chargé de la section des chemins de fer et plus particulièrement du directeur des transports militaires de la zone de l'intérieur.

\section{L'effort militaire et industriel}

22 L'incidence du conflit sur la toile ferroviaire se fait fait sentir immédiatement, comme le souligne l'ingénieur en chef de l'exploitation de la Compagnie PLM en 1916:

«Le nombre des embranchements particuliers sur le réseau a connu une augmentation de 63 embranchements ou sous-embranchements nouveaux, alors que la progression n'avait été moyennement que de 13 pour les huit dernières années, pourtant prospères ${ }^{31}$. "

L'administration de la Guerre est bien entendu l'un des principaux initiateurs de ce renouveau. Entre 1915 et 1919, année du rétablissement de l'autonomie de gestion des réseaux, la compagnie ouvre 58 embranchements au profit des autorités militaires françaises, près de $30 \%$ des ouvertures effectuées durant cette période. Dès 1916 d'importants établissements, comme les ateliers de chargement d'obus à Moulins ou Vénissieux ou les nouvelles usines du service des poudres dans le Midi, sont mis en exploitation afin de faire face à des besoins munitionnaires croissants. Suite à l'arrivée en France des troupes américaines en juin 1917, la compagnie doit composer avec les besoins d'une nouvelle administration et, durant les années 1918 et 1919, le réseau met en service 11 embranchements particuliers pour l'autorité militaire américaine. Sont desservis notamment les très importants centres d'hospitalisation de Beaune, Allerey, Mesves-Bulcy et Mars.

Néanmoins les installations militaires ne sont pas les uniques bénéficiaires de ce dispositif de desserte. Dans une logique de guerre totale, de grandes usines se mettent au service de la défense nationale et se raccordent au réseau PLM. On relève 62 ouvertures de ce type entre 1915 et 1918, soit $38 \%$ du nombre total de mises en service. Il faut également noter qu'entre 1916 et 1918 la compagnie établit 12 embranchements pour l'administration des Ponts et Chaussées, en vue de l'exploitation de carrières et de l'expédition de macadam destiné aux routes de la zone des armées. Au total, entre 1915 et 1919 , près des trois quarts des voies ouvertes par la Compagnie PLM sont destinés aux autorités militaires ou à des établissements et des administrations œuvrant pour la défense nationale. Au moyen de ces raccordements, le réseau PLM contribue pour une grande part à l'effort de guerre.

Par ailleurs, conformément aux accords de 1915 fixant un tarif d'exploitation de base, la compagnie perçoit une somme pour loyers de wagons, pénalités et frais de manœuvre sur les embranchements militaires. Compris dans les «transports de guerre ", ces paiements visent à amortir les frais de fonctionnement des raccordements particuliers. En 1915, la compagnie perçoit 243700 francs à ce titre et, au fil du conflit, un certain nombre d'heures de manœuvre est payé par l'administration de la Guerre. $\mathrm{Du}$ fait de ces embranchements, le réseau récupère chaque année une somme comprenant des arriérés importants dus par les autorités militaires ${ }^{32}$. Comparés aux recettes totales des transports de guerre, les embranchements n'en représentent qu'une part infime, oscillant entre $0,25 \%$ en 1915 et 2,68 \% du total en 1919. Au-delà de l'aspect stratégique et financier, il est également intéressant d'analyser la dimension 
technique des embranchements militaires pour saisir le poids réel du conflit sur le réseau PLM.

\section{Des inconvénients exacerbés}

Dès la mobilisation le réseau est engorgé. Le trafic connaît une croissance immédiate due à l'activité des établissements raccordés. Les principales gares se trouvent dans un état de gêne permanent, provenant du stationnement des wagons des services de la Guerre et des manœuvres nécessitées par la desserte des installations militaires. Cela génère de graves difficultés : retards de trains, formations incomplètes, ceci malgré une augmentation des manœuvres à la machine de $27 \%$ entre 1914 et $1915^{33}$. Cette situation pousse les autorités militaires à se tourner vers d'autres modes de desserte. À Lyon, devant la crise des transports et l'engorgement des gares, les arsenaux se raccordent au réseau de tramways. Durant les années 1916 et 1917, près de 570 tonnes de marchandises transitent sur le réseau urbain de la Compagnie des omnibus et tramways de Lyon à destination du parc d'artillerie ou de l'arsenal de Perrache ${ }^{34}$. Malgré les avantages dont elle bénéficie sur le réseau PLM, l'administration militaire tente donc de s'adapter à la crise et diversifie ses moyens d'approvisionnement. Par ailleurs, l'exploitation des embranchements particuliers devient réellement problématique dans les zones stratégiques. En 1918, la région de Dijon connaît d'importantes difficultés devant le reflux des services des armées et l'afflux des services américains cherchant à se raccorder à un réseau déjà saturé3 ${ }^{35}$.

Hormis les difficultés de circulation propres à tous les trafics, les transports militaires connaissent des risques supplémentaires dus au transit de matières explosives, considérable en temps de guerre. En raison des offensives et de l'importance prise par l'artillerie au cours $\mathrm{du}$ conflit, les transports de munitions augmentent considérablement en 1916 et $1917^{36}$. Ce risque fait l'objet de remarques de la part des administrations locales tolérant la desserte d'établissements militaires ou de défense nationale. Les autorités de l'armée sont également vigilantes. Lors de l'installation d'un dépôt d'explosifs à Lyon, le gouverneur militaire s'inquiète de la proximité de grands établissements et des dispositifs arrêtés pour éviter tout accident lors de leur transport ${ }^{37}$. L'embranchement à destination de ce dépôt sillonne un quartier dans lequel sont situés un parc d'artillerie, une usine à gaz ainsi que d'importants locaux rassemblant près de 8000 soldats. La crainte du gouverneur militaire de Lyon est justifiée au vu des données conservées par l'administration municipale lyonnaise concernant la location de l'embranchement desservant l'usine concernée. Entre janvier 1915 et janvier 1919 circulent près de 56000 tonnes de marchandises tels que des obus, des bombes, des fusées, du matériel pyrotechnique et du matériel d'artillerie lourde, soit $12 \%$ du trafic total de cet embranchement ${ }^{38}$. Si on ne relève aucun accident grave sur les embranchements militaires avant la Première Guerre mondiale, le danger est alors accentué du fait d'un transit important. Le réseau PLM connaît un accident dramatique en 1918. Il s'agit de l'explosion d'un convoi chargé d'obus, destiné à l'embranchement de l'entrepôt de munitions des Gravanches, en stationnement à la gare de triage de Clermont-Ferrand: 133 wagons sont détruits, des immeubles voisins sont endommagés, un soldat est tué et quatre agents sont blessés ${ }^{39}$. Si les accidents de ce type sont rares, les sinistres prennent rapidement des proportions importantes. 

réseau PLM. Le 2 février 1919, un décret restitue la direction des réseaux aux administrations qui en étaient chargées en temps de paix. La Compagnie PLM hérite des multiples embranchements établis pour les besoins militaires durant la guerre, ce qui lui confère un attrait nouveau.

\section{L'impact du conflit sur la toile ferroviaire}

\section{L'héritage structurel du conflit}

Entre 1914 et 1919, le nombre d'embranchements particuliers en service sur le réseau PLM est passé de 552 à $745^{40}$. Ce taux de croissance est l'un des plus forts parmi les grands réseaux français. Si les constructions d'embranchements ralentissent depuis la fin des hostilités, le nombre de ces installations ne cesse de croître. Alors que la compagnie procède à la suppression de voies provisoires établies pour les besoins de la guerre ${ }^{41}$, elle reçoit des demandes visant au maintien des embranchements construits pour les besoins militaires et ceux de la défense nationale. Comme le rappelle Anne Desplantes, il fut envisagé de céder les voies construites pendant le conflit et pouvant présenter une utilité commerciale. Les réseaux reprennent à leur compte près de 1035 kilomètres de voies militaires françaises ${ }^{42}$.

Apparaît le problème du statut à accorder à ces anciennes voies, maintenues à titre d'embranchements particuliers.

«Certains réseaux s'avisèrent que les industriels pouvaient parfaitement être considérés comme des embranchés réguliers, bien que n'exploitant qu'un tronçon de voie ne constituant pas en propre, un embranchement particulier. Mis en demeure d'acquitter les droits d'embranchement, certains acceptèrent, d'autres refusèrent. La question était des plus délicates. Faute de contrat avec ces industriels, le chemin de fer paraissait en assez mauvaise posture pour réclamer ces droits $^{43}$. »

31 La Compagnie PLM semble épargnée. Des conventions sont rapidement établies, visant au maintien des embranchements provisoires établis pendant les hostilités. Entre 1919 et 1926, on relève une quinzaine de traités de ce type. Certains industriels s'étaient même accordés dès 1916 avec la compagnie pour l'exploitation de voies provisoires établies par l'administration des Ponts et Chaussées ${ }^{44}$.

L'effort de guerre réalisé par la Compagnie PLM confère au réseau un attrait industriel nouveau. En dehors des demandes visant au maintien des voies établies durant la guerre, la compagnie fait l'objet de sollicitations dès les années 1918 et 1919, avec 80 et 114 demandes d'embranchements. Si par la suite l'attrait du réseau est moins affirmé, les demandes de raccordement demeurent régulières. On relève une moyenne annuelle de 62 requêtes pour les années 1921, 1922 et 1923. La période d'après guerre souligne donc à son tour le rôle majeur joué par les embranchements particuliers durant le conflit, un dynamisme initié par le ministère de la Guerre.

Cependant, l'essor des embranchements sur le réseau PLM pendant la Première Guerre mondiale ne peut être considéré sans prendre en compte la dimension locale de la desserte militaire. À cet égard la commune de Lyon, principale ville de l'arrière durant la guerre, adopte une politique avantageuse favorisant la desserte ferroviaire des établissements militaires et des diverses usines travaillant pour la défense nationale. La 
municipalité n'exige qu'une faible redevance pour l'occupation du sol public par les voies ferrées et le délai d'autorisation est considérablement réduit. Entre 1915 et 1918, l'atelier de construction de Lyon et le parc d'artillerie établissent 13 raccordements sur des voies de tramways ou de chemin de fer et en 1921 la superficie des voies ferrées exploitées sur le sol communal autour de l'arsenal de Perrache s'élève à $30080 \mathrm{~m}^{2}$.

Aussi l'essor de la desserte particulière dû à la première guerre mondiale permet-il à la municipalité de reconsidérer l'emprise des voies ferrées sur son territoire. Devant les nombreux raccordements à destination d'établissements militaires et d'usines de défense nationale, le conseil municipal de Lyon est amené dès 1920 à statuer sur leur conservation ainsi que sur les redevances à appliquer à l'ensemble des voies particulières de la commune ${ }^{45}$. L'année suivante, les édiles lyonnais s'attachent à régulariser précisément la situation des voies desservant les établissements militaires de la ville ${ }^{46}$. Au-delà du simple rapport avec la Compagnie PLM, l'administration de la Guerre a donc permis, par ses besoins en matière de transport et d'approvisionnement, de reconsidérer la structuration de l'espace urbain lyonnais ainsi que les mesures tarifaires découlant de la desserte particulière. Par ailleurs les exigences des établissements militaires durant la guerre, qui ont amené l'utilisation des voies de tramways, engendrent une réflexion autour des nouveaux modes de transport de marchandises au sein des agglomérations ${ }^{47}$.

L'apport structurel de la Première Guerre mondiale est donc très varié. Le conflit permet au réseau PLM de connaître une certaine attractivité et un développement sans précédent des embranchements. La guerre a également une incidence au niveau de la desserte locale, amenant les municipalités à reconsidérer l'emprise des voies militaires sur leur territoire ainsi que les moyens mis en œuvre pour assurer cette desserte. À partir des années 1920, l'administration de la Guerre dispose d'un dispositif d'approvisionnement densifié et adapté à ses besoins.

\section{Un dispositif de desserte modernisé}

36 Le premier conflit mondial met en exergue les liens existant entre le ministère de la Guerre et les réseaux de chemin de fer. Les rapports entre la compagnie et l'autorité militaire deviennent plus réguliers: 61 projets de traités concernant des embranchements militaires sont approuvés par le conseil d'administration entre 1920 et 1936, contre 45 pour la période qui s'étend de 1862 à 1913. La part des accords conclus avec les ministères de la Guerre, de l'Air et de la Marine augmente entre ces deux périodes, passant de $6,82 \%$ à 9,74\%. Le conflit a donc permis à la compagnie de tisser des liens très étroits avec l'administration militaire, comme en témoigne le nombre moyen de traités approuvés entre 1920 et 1936, entre trois et quatre par an (fig. 2). Ces actes reflètent en partie l'héritage du conflit: deux tiers concernent la régularisation et le maintien de voies ouvertes pour les besoins de l'administration de la Guerre ainsi que des conventions pour l'exploitation d'embranchements établis par l'autorité militaire américaine, dont certaines voies ont été conservées.

Conscient de l'importance du ravitaillement par voie ferrée, le ministère de la Guerre poursuit avec régularité la politique engagée depuis 1872. L'état-major envisage la répartition systématique des établissements militaires le long des réseaux de communications ou à leur proximité. Le conseil d'administration de la compagnie approuve 19 traités de premier établissement entre 1920 et 1934, près d'un par an en 
moyenne. La majorité des embranchements particuliers desservant les grands centres ont été conservés et de nouveaux pôles sont desservis : les services de santé avec la réserve de médicaments de Marseille, le service aéronautique avec le centre d'aviation entre Istres et Miramas, ainsi que de nombreux dépôts et usines. Un embranchement est même établi à Hussein-Dey en Algérie, en 1920. Par un effort d'entretien et de régularisation des voies existantes, le réseau de desserte des établissements militaires est parfaitement structuré à la veille de la Seconde Guerre mondiale. Le ministère de la Guerre compte en 193956 kilomètres d'embranchements établis en pleine voie, près de $15 \%$ du kilométrage des voies raccordées en dehors des gares sur le réseau sud-est ${ }^{48}$. Le premier conflit mondial a donc permis la constitution d'un réseau d'approvisionnement moderne au service de l'administration militaire, tant sur le réseau PLM que sur les autres réseaux ${ }^{49}$.

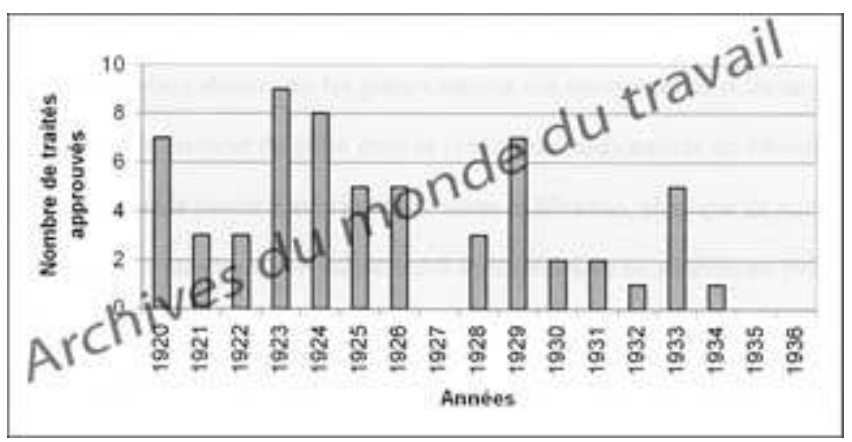

Figure 2. Traités d'embranchement conclus entre la Compagnie PLM et le ministère de la Guerre (1920-1936). Source : procès-verbaux du conseil d'administration de la Compagnie PLM, janvier 1920 décembre 1936, AN (CAMT), 77 AQ 209 à 224.

La Première Guerre mondiale et les nécessités de ravitaillement ont permis à l'étatmajor de concevoir de manière nouvelle le rôle et l'importance des établissements destinés à entretenir et à expédier des ravitaillements. Outre les surfaces nécessaires à l'emmagasinage des stocks, les autorités militaires exigent «un outillage en voies ferrées et quais de chargement suffisant pour effectuer des expéditions par trains complets et pour avoir un débit journalier considérable ${ }^{50}$. Le rail fait désormais partie intégrante des stratégies de développement des établissements dépendant de l'administration militaire. Construit durant le conflit sur une zone extensible, l'arsenal de Roanne répond à ces exigences, identiques à celles de l'industrie moderne. Relié aux lignes de la Compagnie PLM, cet établissement bénéficie d'infrastructures et de matériel de transport dignes des grands groupes industriels ${ }^{51}$.

En adoptant les stratégies de la grande industrie, l'administration militaire s'insère dans le tissu greffé autour du chemin de fer, au sein duquel elle est parfaitement intégrée. Les relations entretenues par le ministère de la Guerre avec les autres usagers des embranchements particuliers soulignent le rôle de cette administration sur le réseau PLM.

\section{Un rôle reconnu par les industriels}

Raccordé au même titre que chaque client du réseau, le ministère de la Guerre est traité par la compagnie à l'instar des autres propriétaires de voies privées. En effet, l'administration militaire ne jouit en période de paix d'aucun avantage, partageant parfois avec les autres embranchés des aiguillages communs. 
41 Après la guerre, les compagnies de chemins de fer se trouvent dans l'obligation de majorer leurs tarifs de transport, afin de faire face à l'augmentation du prix des matières premières et aux conséquences financières désastreuses du conflit. Les ingénieurs de la Compagnie PLM décident de mettre en harmonie les redevances des traités d'embranchement avec les charges financières et techniques que leur desserte impose et les raccordements militaires ne sont pas exemptés. Dans le cas de la ville de Lyon, entre 1922 et 1936, près de $8 \%$ des avenants modificatifs concernent des embranchements de l'armée.

42 Par ailleurs, lors de l'utilisation de soudures communes, l'autorité militaire évite tout litige. L'exploitation en gare de Lyon-Guillotière d'un raccordement commun au parc d'artillerie, à la ville et à la Compagnie du gaz de Lyon illustre les relations exemplaires entretenues par le ministère de la Guerre avec les autres embranchés. Cette attitude est saluée lors de l'anniversaire de l'Association nationale des propriétaires et usagers d'embranchements particuliers, le $1^{\mathrm{er}}$ mai $1938^{52}$. Le représentant de l'armée à la Chambre vient rappeler « l'affection particulière » portée par le ministère de la Guerre aux propriétaires d'embranchements.

43 La signification du rapport entre l'armée et les industriels embranchés est essentielle au vu du potentiel technique et structurel mis à disposition par les militaires depuis le conflit. À Lyon, le conseil municipal se félicite en $1921 \mathrm{du}$ fait que l'atelier de construction mette son réseau de voies privées au service du commerce local mais aussi de la ville ${ }^{53}$. Malgré leur nature spécifique, les embranchements militaires peuvent donc assurer un usage commun. Les propriétaires d'embranchements envisagent l'association d'infrastructures telles que les quais militaires à l'exploitation commerciale et industrielle de leurs voies, de façon à ne pas laisser «incultes » des installations de l'armée ${ }^{54}$. Ces infrastructures offrent de nouvelles possibilités d'exploitation dans des zones souvent saturées ${ }^{55}$. Le rapport entre les réseaux et le ministère de la Guerre se trouve ainsi modifié. Depuis le conflit, les industriels cherchent à tirer profit de la présence militaire sur le réseau et de son dynamisme en matière de chemin de fer, conférant à cette administration un statut particulier parmi les clients de la Compagnie PLM.

44 Les ministères de la Guerre et de la Marine engagent au cours de la seconde moitié du $\mathrm{XIX}^{\mathrm{e}}$ siècle un partenariat privilégié avec les réseaux de chemins de fer. Le nombre d'embranchements particuliers à destination des établissements militaires connaît un essor important, qui fait de l'armée un client majeur de la Compagnie PLM. Si elle tarde à reconnaître l'importance d'une desserte efficace, suite au choc de 1870 l'administration de la Guerre engage une politique innovante, permettant la constitution d'un réseau d'établissements autour des lignes de chemin de fer. Le premier conflit mondial permet le développement de ce tissu de desserte et la constitution d'un dispositif d'approvisionnement moderne et adapté, que les autorités militaires s'évertuent à entretenir et à améliorer durant l'entre-deux-guerres. Le tissu d'embranchements militaires confère alors un certain dynamisme au réseau PLM dont bénéficient les autres usagers, tant par le nombre de voies en service que sur le plan technique. L'essor de la desserte militaire est également sensible au niveau local, permettant de reconsidérer le mode d'insertion ferroviaire dans l'espace urbain. Les établissements des ministères de la Guerre et de la Marine s'agrègent ainsi au tissu industriel existant autour des lignes de chemin de fer du réseau PLM et calquent leur fonctionnement sur l'industrie, en élaborant des logiques d'implantation et de 
développement rationalisées autour du rail. À travers l'exemple des embranchements militaires, il faut donc considérer la place du chemin de fer tant dans l'activité nationale que dans la structuration de l'espace régional et urbain. À la veille de la Seconde Guerre mondiale, un véritable réseau stratégique et industriel a pris forme sur l'ensemble du territoire.

\section{NOTES}

1. - Vincent DELVERT, «Aspect du rôle des cinq grandes compagnies de chemins de fer pendant la guerre de 1870-1871 », maîtrise d'histoire, université de Paris-Sorbonne (Paris IV), 1982 ; Anne DESPLANTES, «Les grands réseaux de chemin de fer français pendant et après la Première Guerre mondiale 1914-1921 ", thèse d'histoire, université Paris X-Nanterre, 1997, 754 p.

2. - Nous pouvons citer le travail de Pascal Puig, même s'il ne mentionne pas la Compagnie PLM. Pascal PUIG, «L'armée française et les chemins de fer, des origines à 1954 » mémoire de DEA d'histoire, université Paul Valéry-Montpellier III, 1992, 127 p.

3. - Raymond GODFERNAUX, "Les embranchements industriels et leur utilité ", Revue générale des chemins de fer (désormais RGCF), mars 1922, p. 347-374 et février 1931, p. 225-252 ; André CHALE et Gaston CHATEL, Les Embranchements particuliers : étude juridique, théorique et pratique, Paris, Dalloz, 1930, $245 \mathrm{p}$.

4. - Le lieutenant-colonel Andriot consacre une partie de sa « Note sur les chemins de fer de la Ruhr ", aux embranchements industriels, RGCF, 4e année, 2e semestre, $\mathrm{n}^{\circ}$ 2, août 1922, p. 95-96.

5. - François CARON, «Histoire de l'exploitation d'un grand réseau français, la Compagnie du chemin de fer du Nord de 1846 à 1936 ", thèse pour le doctorat d'État, Faculté des Lettres de Paris-Nanterre.

6. - Ève ROUSSEAU, « Dynamiques du "porte à porte" ferroviaire. L'embranchement particulier de 1947 à 1999 », maîtrise de géographie, université Paris X-Nanterre, 2000.

7. - Jean-Pierre WILLIOT, «De l'influence des chemins de fer sur l'industrie du gaz au xixe siècle ", in Dominique BARJOT et Michèle MERGER (dir.), Les Entreprises et leurs réseaux: hommes, capitaux, techniques et pouvoirs XIXe-XXe siècles. Mélanges en l'honneur de François Caron, Paris, Presses de l'université de Paris-Sorbonne, 1998, p. 659-669.

8. - Consultables au Archives nationales, Centre des archives du monde du travail (CAMT), les procès-verbaux du conseil d'administration de la Compagnie PLM sont conservés d'avril 1862 à décembre 1936 (77 AQ 180 à 224). Les répertoires des années 1857 à 1862 sont conservés de manière éparse et lacunaire au sein des archives des compagnies fusionnées, ce qui fausse toute étude partant de 1857.

9. - Les embranchements particuliers militaires de la Compagnie PLM ne font pas l'objet d'un classement particulier, contrairement aux dossiers conservés sur le même thème par le Syndicat des chemins de fer de petite ceinture par exemple.

10. - Déclaration du général Lamarque en 1832, mentionnée in SIMONNET (commandant), «Les débuts militaires du chemin de fer entre 1827 et 1870 ", Bulletin spécial du centenaire, 1895-1995, $\mathrm{n}^{\circ} 265$ (octobre 1995), p. 45, revue de l'ANOST, Association nationale des officiers spécialistes des transports militaires. 
11. - L'arsenal royal de Woolwich est desservi par une voie de tramway à traction animale dès 1825. Jack SIMMONS, The Railway in Town and Country, 1830-1914, Londres, Newton Abbot, 1986, p. 35.

12. - François-Prosper JACQMIN, Les Chemins de fer pendant la guerre de 1870-1871. Leçons faites en 1872 à l'École des Ponts et Chaussées, Paris, Hachette, 1872, p. 47.

13. - Alfred-Auguste ERNOUF, Histoire des chemins de fer français pendant la guerre franco-prussienne, rééd., Cavalière, Éditions du Layet, 1980, p. 448.

14. - Ibid., p. 334-335.

15. - F.-P. JACQMIN, Les Chemins de fer..., op. cit., p. 349.

16. - Archives de la chambre de commerce et d'industrie de Lyon, S4 TC3 5, pétition adressée par les habitants du quartier de Perrache au ministre de l'Agriculture, du Commerce et des Travaux publics, le 20 novembre 1859, concernant la réouverture de la gare de Perrache au trafic de petite vitesse.

17. - François CROUZET, « Remarques sur l'industrie des armements en France (du milieu du xixe siècle à 1914)", Revue historique, $\mathrm{n}^{\circ} 510$ (avril-juin 1974), p. 409-422.

18. - AN (CAMT), 77 AQ 184, conseil d'administration de la Compagnie PLM, séance du 4 octobre 1872.

19. - Raymond GODFERNAUX, Louis MARLIO, E. MAZERAT et Louis-Pierre-Joseph VERGNIAUD, Voies ferrées (France, Algérie, Tunisie et Colonies françaises), Paris, P. Dupont, 1912, tome 2, p. 32.

20. - On ne recense en 1911 que neuf embranchements militaires en service sur le réseau du Nord, la plupart ouverts avant 1892, AN, Centre historique des Archives nationales (CHAN), F14 15346. État des embranchements particuliers raccordés au réseau du Nord à la date du 1er juillet 1911.

21. - Archives municipales de Lyon (AML), II 221, notice historique sur l'arsenal, p. 49.

22. - Ibid., p. 95.

23. - Archives départementales du Rhône (ADR), $104 \mathrm{~W}$ 441, avis de la commission mixte des travaux publics concernant l'établissement d'un embranchement particulier destiné à relier la fabrique de produits chimiques Coignet avec la gare de Vénissieux, le 20 janvier 1891.

24. - ADR, $104 \mathrm{~W}$ 420, PLM, embranchements, 1870-1890.

25. - AML, II 221, notice..., op. cit., p. 42.

26. - ADR, $104 \mathrm{~W}$ 462, raccordement par la gare de l'est de Lyon de la station-magasin de Villeurbanne et de la gare de la Part-Dieu PLM, 1896.

27. - AN (CAMT), 77 AQ 252, rapport de l'ingénieur en chef de l'exploitation au directeur, annexé à la séance du 3 décembre 1914 de la commission du budget.

28. - Ibid.

29. - AN (CAMT), $77 \mathrm{AQ} 252$, accord des réseaux avec le ministère de la Guerre concernant les conditions d'utilisation des embranchements particuliers par les services militaires pendant la guerre, le 20 octobre 1915, annexé au procès-verbal de la séance du 24 mars 1916 de la commission du budget.

30. - Ibid.

31. - AN (CAMT), 77 AQ 253, rapport de l'ingénieur en chef de l'exploitation au directeur, annexé au procès-verbal de la séance du 5 avril 1917 de la commission du budget.

32. - La Compagnie PLM perçoit pour l'exploitation des embranchements militaires durant les années 1917, 1918 et 1919 près de neuf millions de francs, dont 75 \% d'arriérés en moyenne.

33. - AN (CAMT), 77 AQ 252, rapport de l'ingénieur en chef..., op. cit.

34. - AML, 923 WP 121, emploi du réseau de la Compagnie OTL pour le transport des marchandises, 1915-1923.

35. - Service historique de la Défense (SHD), 16 N 2822, note pour la direction de l'Arrière, le 23 juin 1918.

36. - Anne DESPLANTES, « Les grands réseaux... », thèse citée, p. 168. 
37. - AML, 945 WP 085 8, général Ebener gouverneur militaire de Lyon au lieutenant-colonel inspecteur des forges le 29 juin 1916, concernant l'installation d'un atelier de pyrotechnie dans l'usine de la société d'éclairage électrique.

38. - AML, 937 WP 136, exposition internationale urbaine de 1914, abattoirs de la Mouche, location d'un emplacement et de l'accès à la voie ferrée particulière, 1915-1919.

39. - SHD, $16 \mathrm{~N}$ 2822, rapport en date du 24 juillet 1918, du chef de bataillon de Montrachy, commissaire militaire de la sous-commission de réseau de Clermont-Ferrand, au sujet d'un incendie sur les voies de triage de la gare de Clermont-Ferrand le 19 juillet 1918.

40. - Raymond GODFERNAUX, « Les embranchements industriels... », art. cité, février 1931, p. 244. 41. - La compagnie supprime en 1919, 1922 et 1923 respectivement cinq, six et deux embranchements particuliers.

42. - Anne DESPLANTES, thèse citée, p. 596.

43. - André CHALE et Gaston CHATEL, Les Embranchements particuliers..., op. cit., p. 56-61.

44. - Si nous relevons quatre traités en 1916, deux en 1917 et deux en 1918, les données fournies par le conseil d'administration pour la période 1913-1919 ne sont pas exhaustives. Elles permettent néanmoins de dresser un aperçu de la politique engagée par la compagnie PLM en vue du maintien des embranchements établis pendant la guerre.

45. - Conseil municipal de Lyon, séance du 2 août 1920, voies ferrées particulières établies sur le domaine public, fixation d'un tarif de redevances.

46. - Conseil municipal de Lyon, séance du 17 octobre 1921, voies ferrées de l'atelier de construction, abaissement de la redevance.

47. - « Camionnage par les voies de tramways », Le Génie civil, 7 septembre 1918, p. 192-194.

48. - AN (CHAN), F14 17490. SNCF, liste des embranchements particuliers dont l'origine est située entre deux gares, 1939.

49. - À titre de comparaison, tous les embranchements exploités par le ministère de la Guerre autour de la grande ceinture en 1930, constituant $18 \%$ des voies en service, ont été établis durant les années 1914 et 1915, Bruno CARRIÈRE et Bernard COLLARDEY, L'Aventure de la Grande Ceinture, Paris, La Vie du rail, 1993, p. 306.

50. - Ministère de la Guerre, Instruction provisoire sur l'organisation des communications et des transports militaires en temps de guerre. Annexe $n^{\circ} 6$ à l'instruction provisoire sur l'emploi tactique des grandes unités, Paris, Imprimerie nationale, 1922, p. 27.

51. - Michel BARRAS, Histoire de l'arsenal de Roanne: 1916-1990, Lyon, Éditions lyonnaises d'art et d'histoire, 1998, p. 49.

52. - AN (CHAN), F14 17490, discours de M. Thouvenin, président de l'Association nationale des propriétaires et usagers d'embranchements particuliers, le 1er mai 1938, à l'occasion du 10e anniversaire de la fondation de l'association.

53. - Conseil municipal de Lyon, séance du 17 octobre 1921, voies ferrées de l'atelier de construction..., doc. cité.

54. - AN (CHAN), F14 17490, discours de M. Thouvenin..., doc. cité.

55. - Deux industriels sont autorisés par la Compagnie PLM à exploiter des embranchements constitués par des voies de quais militaires, en 1921 et 1924. 


\section{AUTEUR}

\section{THOMAS BOURELLY}

Doctorant en histoire contemporaine, université Lumière-Lyon 2 (thèse soutenue par l'AHICF) 